\title{
Thermal and Thermal Stress Analysis of a Thin-Film Thermoelectric Cooler Under the Influence of the Thomson Effect
}

\author{
Mei-Jiau Huang ${ }^{1}$, Po-Kuei Chou ${ }^{1}$, and Ming-Chyuan $\operatorname{Lin}^{2}$ \\ ${ }^{1}$ Mechanical Engineering Department, National Taiwan University,Taipei,Taiwan \\ ${ }^{2}$ National Synchrotron Radiation Research Center, Shinchu, Taiwan \\ mjhuang@ntu.edu.tw, (886)233662696
}

\begin{abstract}
This work has two parts. The first part details the thermal analysis of a thin-film thermoelectric cooler under the influence of the Thomson effect, the Joule heating, and the Fourier's heat conduction. A constant Thomson coefficient, instead of traditionally a constant Seebeck coefficient, is assumed. The influence of the Thomson effect on the cooling power, maximum temperature difference and optimum current density is then explored. A modified thermal conductance and a modified electric resistance are resulted due to the Thomson effect. The second part of this paper performs the analysis of the thermal stresses induced by the temperature differences created by the TE cooler via a non-coupled thermal elastic theory. The results provide a preliminary knowledge to judge whether the thin-film structures fail due to the thermal stresses, especially due to the shear stresses in between two adjacent layers.
\end{abstract}

\section{Introduction}

Thermoelectric coolers have advantages over the traditional heat pumps because they are compact and highly reliable, have no moving parts and use no refrigerants. The poorer cooling efficiency, which is fundamentally limited by the material properties, of thermoelectric devices however limits their applications. A conventional thermal analysis of a thermoelectric cooler usually takes the Fourier's heat conduction, the Joule's heating, and sometimes the radiation and convection heat transfers between the thermoelectric element and the ambient gas into considerations [1-2]. The analysis shows that a material of large Seebeck coefficient $(\alpha)$, high electrical conductivity $(\sigma)$, and low thermal conductivity $(k)$ is desired, namely the figure-of-merit $Z=\sigma \alpha^{2} / k$ is critical for the thermal performance as well as the efficiency.

Nonetheless, a careful examination finds that in the conventional analysis, the temperature dependence of the Seebeck coefficient is ignored, that is the Thomson effect is not concerned [3]. Not impossible is that the reversible Thomson heat has the potential of changing the temperature profiles in the thermoelectric element, modifying the associated thermal conductance and electric resistance, and resulting possibly in a better thermal performance [4]. In the present study, an one-dimensional analysis will be employed to explore the influence of the Thomson effect on the thermal performance of a thin-film thermoelectric cooler like that used by Völklein et al [5]. The induced thermal stresses will be investigated also via a non-coupled elastic theory. Above all, the induced shear stresses in between two adjacent thin-film layers may tear the thin-film structures and will be analyzed also.

\section{Thermal Analysis}

Figure 1 shows the cross-sectional view of a single thinfilm thermocouple, including the supporting membrane, $p$ type and n-type thermoelectric films that are separated by an insulating layer. In order to highlight the Thomson effect, the radiation and convection heat transfer between the thermocouple and the ambient gas will be disregarded. According to the nonequilibrium thermodynamics, the steady temperature distribution $T(x)$ is governed by

$$
0=\frac{d}{d x}\left((k A)_{t} \frac{d T}{d x}\right)+\frac{I^{2}}{(\sigma A)_{p n}}-\beta_{p N} I \frac{d T}{d x}
$$

where $(k A)_{t} \equiv k_{p} A_{p}+k_{i} A_{i}+k_{n} A_{n}+k_{m} A_{m}, 1 /(\sigma A)_{p n} \equiv 1 / \sigma_{p} A_{p}+1 / \sigma_{n} A_{n}$; $I$ is electric current, $A$ is the cross-sectional area of each layer, $L$ is the length of the thermocouple, and $\beta_{p n}=\beta_{p}-\beta_{n}$ is the Thomson coefficient difference which is assumed constant herein. The subscripts $m, p, n$, and i represent the membrane, p-type, n-type, and the insulating layer respectively. Terms on the right-hand-side of Eqn.(1) are the net axial Fourier's conduction heat transfer rate, the Joule's heat generation rate, and the Thomson heat generation rate, respectively. The solution is found to be

$$
\frac{T(x)-T_{c}}{T_{h}-T_{c}}=(1-\zeta) \cdot \frac{1-\exp \left(\xi \frac{x}{L}\right)}{1-\exp (\xi)}+\zeta \frac{x}{L}
$$

where $\xi \equiv \beta_{p n} I / K_{t}$ and $\zeta \equiv I R_{r} / \beta_{p n}\left(T_{h}-T_{c}\right)$ are the ratio of the associated Thomson heat to the Fourier's conduction heat and the ratio of the Joule's heat to the Thomson heat; $K_{t} \equiv(k A)_{t} / L$ and $R_{t} \equiv L /(\sigma A)_{p n}$ are the "intrinsic" thermal conductance and electric resistance of the thermocouple; $T_{c}$ and $T_{h}$ are the temperatures at the cold junction and the hot junction.

The Fourier's conduction heat rate at the cold junction can now be computed and the heat transfer rate at the junctions become

$$
q_{c}=\alpha_{p n}\left(T_{c}\right) I T_{c}-\left(\eta_{K} K_{t}\right)\left(T_{h}-T_{c}\right)-\left(\eta_{R} R_{t}\right) I^{2} / 2
$$

and

$$
q_{h}=\alpha_{p n}\left(T_{h}\right) I T_{h}-\left(\eta_{K}+\xi\right) K_{t}\left(T_{h}-T_{c}\right)+\left(2-\eta_{R}\right) R_{t} I^{2} / 2
$$

where $\alpha_{p n}=\alpha_{p}-\alpha_{n}$. The effective thermal conductance and the effective electric resistance modified by the Thomson effect can thus be defined as $\eta_{k} K_{r}$ and $\eta_{R} R_{r}$. The modification factors are

$$
\eta_{K}(\xi)=\frac{\xi}{e^{\xi}-1}
$$




$$
\eta_{R}(\xi)=\frac{2}{\xi}-\frac{2}{e^{\xi}-1}
$$

and are shown in Fig.2. As seen, a proper choice of the Thomson coefficient can efficiently reduce the thermal conductance as well as the electric resistance, and consequently can improve the thermal performance of the thermoelectric cooler. From Eqns. (3) and (4), it is also seen that the Fourier's conduction heat at the cold junction is less than that at the hot junction by an amount of Thomson heat $\beta_{p n} I\left(T_{h}-T_{c}\right)$ and the fraction of the Joule's heat flowing to the cold junction is adaptable by varying the Thomson coefficient.

The maximum achievable temperature difference $\Delta T_{\max }$ and the maximum allowable heat load $q_{c, \text { max }}$ can now be derived and shown as

$$
\begin{aligned}
Z_{c} T_{c} & =\frac{\alpha_{p n}}{\beta_{p n}}\left(1-e^{-\xi_{o p t . T}}\right) \\
Z_{c} \Delta T_{\max } & =\frac{\alpha_{p n}^{2}}{\beta_{p n}^{2}}\left\{\xi_{\text {opt.T}}+\exp \left(-\xi_{\text {opt.T }}\right)-1\right\}
\end{aligned}
$$

and

$$
\begin{aligned}
Z_{c} T_{c} & =\frac{\alpha_{p n}}{\beta_{p n}}\left(1+\frac{\xi_{\text {opt.q }}^{2} \exp \xi_{\text {opt.q }}}{\left(1-\exp \xi_{\text {opt.q }}\right)^{2}}+\frac{2 \xi_{\text {opt.q }}}{1-\exp \xi_{\text {opt.q }}}\right) \\
Z_{c} q_{c, \max } & =K_{t} \cdot \frac{\alpha_{p n}^{2}}{\beta_{p n}^{2}} \cdot \frac{\xi_{\text {opt.q }}^{2}}{\left(1-\exp \xi_{\text {opt.q }}\right)}\left(1+\frac{\xi_{\text {opt.q }} \exp \xi_{\text {opt.q }}}{1-\exp \xi_{\text {opt.q }}}\right)
\end{aligned}
$$

where $Z_{c} \equiv \alpha_{p n}^{2} / K_{t} R_{t}$ (figure-of-merit) is evaluated at the cold-junction temperature $T_{c}$.

Figure 3 shows the optimum operating current density $\left(j_{\text {opt.T }}\right)$ and the maximum achievable temperature difference for $A_{i+m}=0.2 \mathrm{~mm} \times 3 \mu \mathrm{m} \times 2, A_{p, n}=0.2 \mathrm{~mm} \times 10 \mu \mathrm{m}$, and $L=0.3 \mathrm{~mm}$ with properties evaluated at $T_{c}=250 \mathrm{~K}$ [5]. As seen the maximum achievable temperature difference is increased by a positive value of $\beta_{p n}$. It is about $42.8 \mathrm{~K}$ when $\beta_{p n}=0$ and is increased to about $57.5 \mathrm{~K}$ when $\beta_{p n}=400 \mu \mathrm{V} / \mathrm{K}$. This is because the current absorbs heat not only at the cold-junction through the Peltier effect but also all the way along the thermoelectric element due to the Thomson effect (positive $\beta_{p n}$ and positive temperature gradients everywhere). The amounts of Joule's heat and Fourier's conduction heat are consequently decreased by factors of $\eta_{R}$ and $\eta_{K}$ respectively. Figure 4 on the other hand shows the maximum allowable heat load for a TE element with a same geometry but properties evaluated at $T_{c}=T_{h}=293 \mathrm{~K}$. The maximum allowable heat load is about $17.8 \mathrm{~mW}$ as $\beta_{p n}=0$ and is increased to be about $19.4 \mathrm{~mW}$ when $\beta_{p n}=400 \mu \mathrm{V} / \mathrm{K}$.

\section{Thermal Stress Analysis}

A one-dimensional elastic stress analysis is adopted herein. It is assumed that the thermal displacement mainly occurs in the $\mathrm{x}$-direction and its variation across the thickness direction is much smaller than that in the $\mathrm{x}$-direction. Non- elastic effects such as plasticity and creep are not taken into consideration. The thermoelectric element is assumed to be clamped at both junctions and free at the top and bottom surfaces. Consequently, a force balance results in

$$
\frac{d^{2} u}{d x^{2}}=\gamma_{0} \frac{d T}{d x}
$$

with

$$
\gamma_{0}=\frac{A_{p} E_{p} \gamma_{p}+A_{i} E_{i} \gamma_{i}+A_{n} E_{n} \gamma_{n}+A_{m} E_{m} \gamma_{m}}{A_{p} E_{p}+A_{i} E_{i}+A_{n} E_{n}+A_{m} E_{m}}
$$

where $u$ is the x-displacement, $E$ is the Young's modulus and $\gamma$ is the thermal expansion coefficient. The solution can now be easily found to be

$$
\frac{u(x)}{L \gamma_{0} \Delta T}=\frac{\zeta}{2} \cdot \frac{x}{L}\left(\frac{x}{L}-1\right)+\frac{1-\zeta}{\xi}\left(\frac{1-\exp \xi \frac{x}{L}}{1-\exp \xi}-\frac{x}{L}\right)
$$

The thermal normal stress is related to the displacement by

$$
\sigma_{x x}=E\left(\frac{d u}{d x}-\gamma\left(T-T_{f s}\right)\right)
$$

where $T_{f s}$ is the free-stress temperature. By substituting Eqn.(12) into Eqn.(13), one can rewrite the thermal normal streass as

$$
\sigma_{x x}=E\left\{\gamma_{0}\left(T-T_{0}\right)-\gamma\left(T-T_{f s}\right)\right\}
$$

where $T_{0}=\phi T_{h}+(1-\phi) T_{c}$ and

$$
\phi=\frac{1-\zeta}{1-\exp \xi}+\frac{1-\zeta}{\xi}+\frac{\zeta}{2}
$$

Equation (14) implies that the thermal stress within each layer can be divided into two parts - one induced by the temperature changes and the other caused by the layered structure. The temperature $T_{0}$ serves as the reference temperature at which the "four-layered structure" is stressfree.

Figure 5 shows the normal-stress distributions along the thermoelectric element that is operated at the optimum current calculated as shown in Fig.4. The thermo-mechanical properties of semiconductor $\mathrm{Si}_{0.5} \mathrm{Ge}_{0.5}$ are employed for the $\mathrm{p}$ type as well as n-type thin films $(E=160 \mathrm{Gpa}$ and $\left.\gamma=4.2 \times 10^{-6} / K\right)$ and those of $\mathrm{SiO}_{2}$ are used for the membrane and insulating thin films $\left(E=75 \mathrm{Gpa}\right.$ and $\left.\gamma=5 \times 10^{-7} / K\right)$. The free stress temperature $\left(T_{f_{s}}\right)$ is set at $293 \mathrm{~K}$. As seen, when the Thomson effect increases, so is the absolute normal stress. All the calculated normal stresses are under the yielding stresses of the materials in use.

The shear stresses in between two adjacent layers can be found now by taking a force balance across each layer. The results, with free top and bottom surfaces, are found to be

$$
\begin{aligned}
\tau_{m, n} & =-E_{m} h_{m}\left(\gamma_{0}-\gamma_{m}\right) \frac{d T}{d x} \\
\tau_{n, i} & =-\left[E_{n} h_{n}\left(\gamma_{0}-\gamma_{n}\right)+E_{m} h_{m}\left(\gamma_{0}-\gamma_{m}\right)\right] \frac{d T}{d x}
\end{aligned}
$$




$$
\tau_{i, p}=E_{p} h_{p}\left(\gamma_{0}-\gamma_{p}\right) \frac{d T}{d x}
$$

The analysis shows that the maximum shear stresses occur at the location with maximum temperature gradient. In addition, the larger the heterogeneity in the thermal expansion coefficients of materials, the larger the shear stress is resulted. Figure 6 shows the shear-stress distributions corresponding to the normal-stress distributions in Fig.5. Because of the materials used, the shear stress distribution along the interface between the p-type layer and the insulating layer is as the same as that along the interface between the membrane layer and the n-type semiconductor. Moreover, the shear stress along the interface between the n-type semiconductor and the insulating layer vanishes. Therefore, only the nonzero distribution is plotted. Due to the Thomson effect, the current absorbs heat near the cold junction and releases heat near the hot junction, causing larger temperature gradients and thus larger shear stresses near the hot junction.

\section{Conclusions}

The thermal performance of a thin-film thermoelectric cooler under the influence of the Thomson effect is investigated. For those cases that the Thomson effect is important, two dimensionless influence parameters - the ratio of the Thomson heat to the conduction heat and the ratio of Joule's heat to the Thomson heat, determine the thermal performance. The fractions of the conduction heat and the Joule's heat that flow back to the cold junction can be significantly reduced when materials with proper Thomson coefficients are selected. Larger maximum achievable temperature differences and larger maximum allowable heat loads are obtainable, providing that the employed p-type semiconductor material has a larger Thomson coefficient than the n-type one. The normal stress distributions within the thermoelectric element as well as the shear stress distributions along the interfaces between two adjacent layers are also analyzed by employing a non-coupled elastic theory. As the thermal performance is improved by the Thomson effect, the induced thermal stresses are increased as well. The computed normal stresses nonetheless are still less than the bulk yielding stresses of the materials in use. Finally, the larger the heterogeneity in the thermal expansion coefficients of materials, the larger the shear stresses are resulted.

\section{Acknowledgments}

The support of this work by the National Science Council, Taiwan, R.O.C. under contract NSC 92-2212-E-002-047 and ITRI under the contract 3000017521 are gratefully acknowledged.

\section{References}

1. D.M. Rowe, CRC Handbook of thermoelectrics, CRC Press LLC, 1995.

2. C. LaBounty, A. Shakouri, J.E. Bowers, "Design and characterization of thin film microcoolers," J. Appl. Phys., Vol. 89 (2001), pp. 4059-4064.

3. J. Chen, Z.Yan, L. Wu, "The influence of Thomson effect on the maximum power output and maximum efficiency of a thermoelectric generator," J. Appl. Phys., Vol. 79 (1996), pp. 8823-8828.
4. M.J. Huang, R.H. Yen, and A.B. Wang, "The influence of the Thomson effect on the performance of a thermoelectric cooler," Inter. J. Heat Mass Transfer Vol. 48 (2005), pp. 413-418.

5. F. Völklein, Gao Min, D.M. Rowe, "Modelling of a mircoelectromechanical thermoelectric cooler," Sensors and Actuators, Vol. 75 (1999), pp. 95-101.

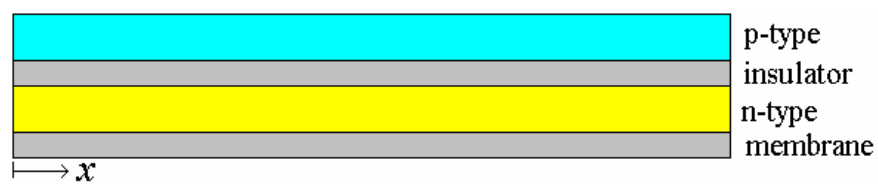

Figure 1: The cross-sectional view of a single thin-film thermocouple.

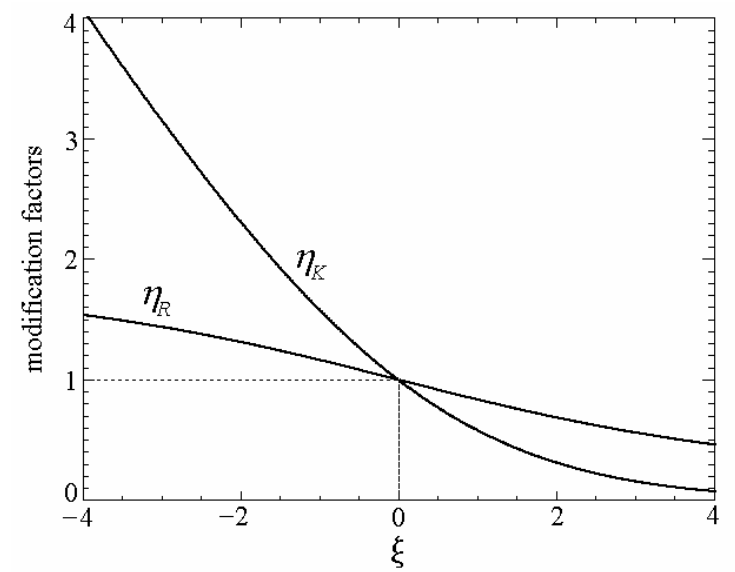

Figure 2: The modification factors of the thermal conductance and the electric resistance due to the Thomson effect.

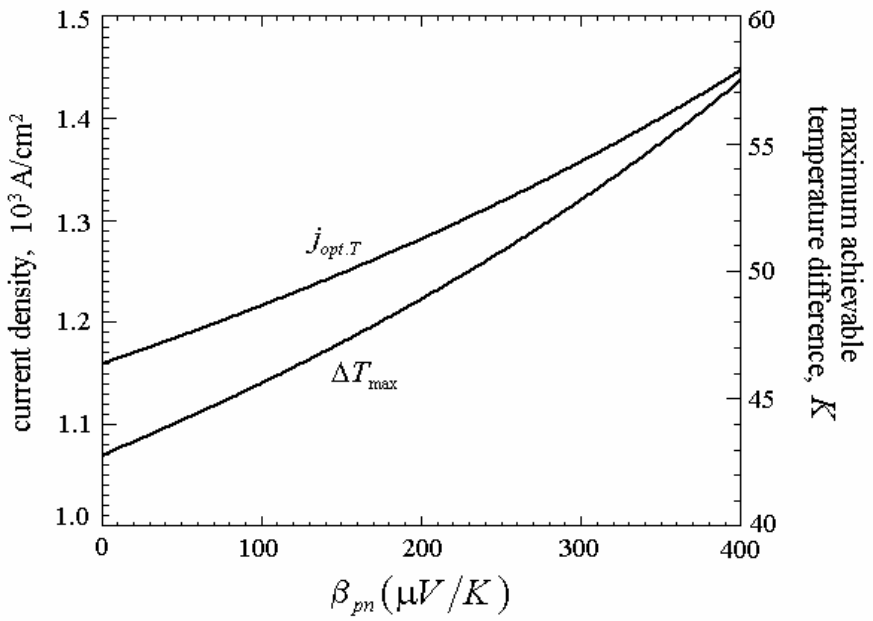

Figure 3: The influence of Thomson effect on the maximum achievable temperature difference and its corresponding optimum operating current density. 


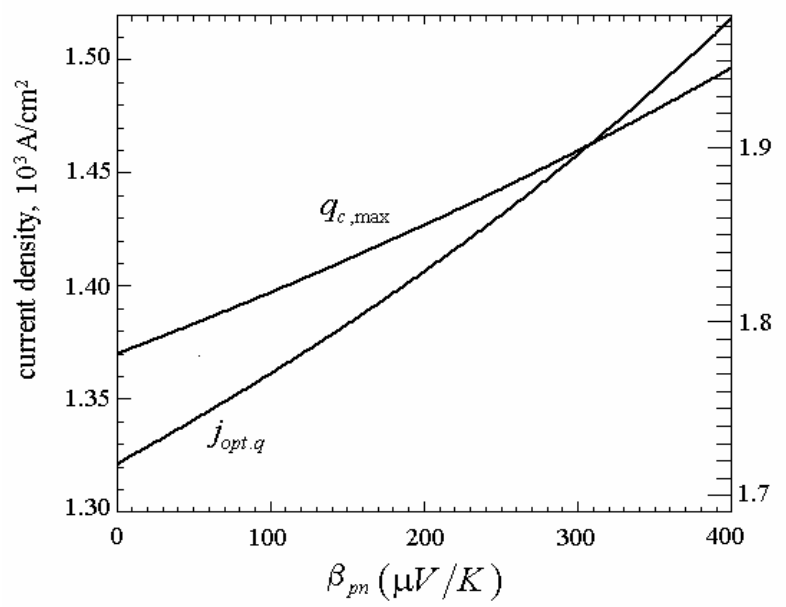

Figure 4: The influence of Thomson effect on the maximum allowable heat load and its corresponding optimum operating current density.
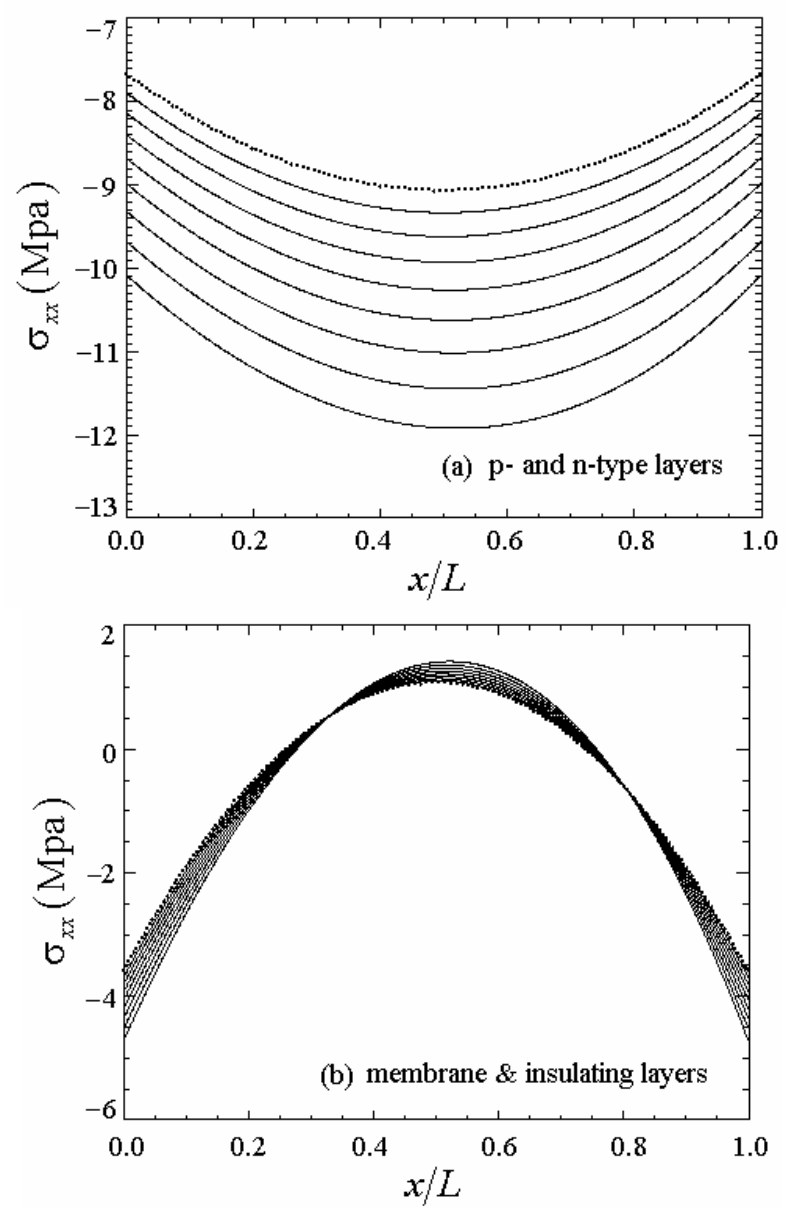

Figure 5: The normal stress distributions of the thermoelectric cooler operated at the optimum currents shown in Fig.5, where $\Delta \beta=50 \mu \mathrm{V} / \mathrm{K}$ and $\beta_{\max }=400 \mu V / K$. The dotted curve is the one corresponding to $\beta_{p n}=0$.

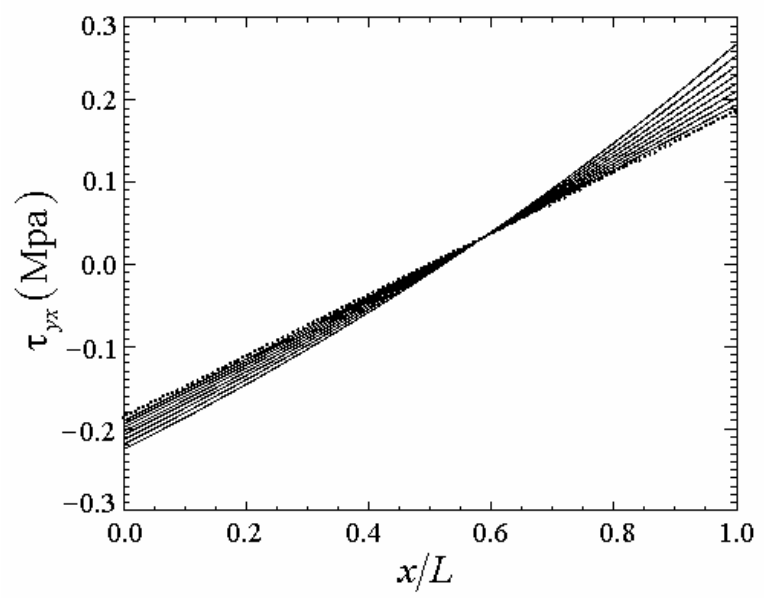

Figure 6: The shear stress distributions along the interface between the insulating layer and the p-type semiconductor layer of the thermoelectric cooler operated at the optimum currents shown in Fig.4, where $\Delta \beta=50 \mu \mathrm{V} / \mathrm{K}$ and $\beta_{\max }=400 \mu \mathrm{V} / \mathrm{K}$. The dotted curve is the one corresponding to $\beta_{p n}=0$. 